\title{
Deformation and Depinning of Superconducting Vortices from Artificial Defects: A Ginzburg-Landau Study
}

\author{
D. J. Priour $\mathrm{Jr}^{1,2}$ and H. A. Fertig ${ }^{1}$ \\ ${ }^{1}$ Department of Physics and Astronomy, University of Kentucky, Lexington, KY40506-0055 \\ ${ }^{2}$ Center for Computational Sciences, University of Kentucky, Lexington, KY40506-0045
}

(October 29, 2018)

\begin{abstract}
Using Ginzburg-Landau theory, we have performed detailed studies of vortices in the presence of artificial defect arrays, for a thin film geometry. We show that when a vortex approaches the vicinity of a defect, an abrupt transition occurs in which the vortex core develops a "string" extending to the defect boundary, while simultaneously the supercurrents and associated magnetic flux spread out and engulf the defect. Current induced depinning of vortices is shown to be dominated by the core string distortion in typical experimental situations. Experimental consequences of this unusual depinning behavior are discussed.
\end{abstract}

PACS numbers: 74.20.-z, 74.60.Ge, 74.76.-w, 68.55.Ln, 61.46.+w

\section{INTRODUCTION}

One of the most important properties of superconductors is their ability to carry currents without dissipation, allowing them to generate large magnetic fields. Many superconductors allow fields to penetrate in bundles of quantized magnetic flux, with associated whirlpools of current known as vortices. When these vortices are mobile, they spoil the perfect conductivity that make superconductors so useful. The quest to increase the maximum dissipationless current $J_{c}$ that a superconductor may carry has thus fueled intense study of vortex pinning.

In recent years, pinning environments of artificially fabricated nanoscale defect arrays have been developed in hopes of better understanding and improving the pinning properties of superconductors. Some of the earlier contributions have involved macroscopic measurements (e.g. $J_{c}$, magnetization) performed on periodic "antidot" arrays ${ }^{1-3}$. The antidot regions contain material which is rendered non-superconducting. Pinning behavior may be studied in these periodic systems using various imaging techniques ${ }^{1,4^{-7}}$.

Much of the theoretical work on vortex pinning in defect arrays has employed numerical studies to focus ${ }^{8,9}$ on the behavior of large collections of vortices under the influence of a driving force (supercurrent). Molecular dynamics ${ }^{8,10}$ approaches in particular usually employ simplified pinning potentials, in part to make possible simulations of large numbers of vortices, but also because information about pinning potentials at the microscopic level is simply unavailable. A few studies ${ }^{11,12}$ have focused on energy scales for pinning varying numbers of flux quanta to the defects, as well as defect-vortex potentials as derived from the London equation ${ }^{13,14}$. However, the latter approach does not allow for variation of the Cooper pair density, and in particular cannot correctly treat the vortex core. In the London approach, vortex cores are usually assumed to be rigid in shape, and interactions of vortices with their environments are determined by the core position as well as the distribution of currents ${ }^{13,14}$. Our results demonstrate that the vortex core in fact deforms dramatically near an artificial defect: when the vortex center is sufficiently close to the defect, a string of suppressed order parameter develops from the vortex position to the pinning center edge, while the currents and magnetic flux spread out over a large area (see Fig. 2 below).

Usually, experimental studies of depinning are conducted in the presence of a net current across the superconducting film. The current exerts a Lorentz force on the flux quantum, dislodging the vortex from the defect for currents exceeding the depinning threshold. We find that, in addition to this, the external current distorts the conservative part of the pinning force for a sufficiently large net current, leading to important changes in the order parameter, supercurrents, and depinning threshold. Specifically, we find for many typical experimental situations that in the presence of a driving current, the core strings associated with specific dots will stretch to reach neighboring dots. At the depinning threshold, the current associated with a vortex unwraps from one pinning site and encircles its neighbor, without the order parameter ever forming a compact core region. Thus, for many experimental situations, interstitial vortices never form when vortices are depinned, as is commonly assumed. The depinning process should instead be understood as one in which the vortex cores are elongated by the driving current, eventually interacting with more than one pinning site at a time and allowing the associated vortex currents to hop from site to site.

\section{METHODS}

Our calculations focus on two dimensional arrays of artificial pinning centers in the form of holes in a bulk superconductor. Our goal is to find the lowest energy state of the system for specified locations of a superconducting vortex; from this we can construct a pinning po- 
tential. The appropriate description of the superconducting state is in terms of Ginzburg-Landau theory, which focuses on a complex superconducting order parameter $\psi(\vec{r})$, for which $|\psi|^{2}$ is proportional to the local density of superconducting electrons. Unlike the London theory, Ginzburg-Landau theory is valid at scales as small as the coherence length, $\xi$. Written in terms of dimensionless variables, the Ginzburg-Landau energy functional is

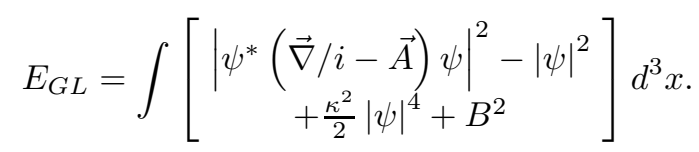

In Eq. 1, $\vec{A}$ is the vector potential, and the magnetic field $\vec{B}(\vec{r})=\vec{\nabla} \times \vec{A} . \quad \kappa \equiv \frac{\lambda}{\xi}$ is the Ginzburg parameter, the ratio of the magnetic penetration depth $\lambda$ and the coherence length. Since the experiments on artificial defect arrays typically involve thin film geometries, we focus on them in our treatment. In considering the thin film limit, it is convenient to integrate by parts and replace the electromagnetic contribution, $|\vec{\nabla} \times \vec{A}|^{2}$ with the quantity $\vec{J} \cdot \vec{A}$. As the thin film limit is approached, one finds that $\psi, \vec{A}$, and $\vec{J}$ vary only very slowly in the $\hat{z}$ direction. The integration in the $\hat{z}$ direction is well-approximated by multiplication by $d$, the thickness of the film, and we may write

$$
E_{G L}=\int\left[\begin{array}{c}
\left|\phi^{*}(\vec{\nabla} / i-\vec{A}) \phi\right|^{2}-|\phi|^{2} \\
+\frac{\lambda_{e f f}}{2}|\phi|^{4}+\vec{j} \cdot \vec{A}
\end{array}\right] d^{2} x .
$$

In Eq. 2, we use the definitions $\phi \equiv d \psi, \vec{j} \equiv d \vec{J}$, and (following standard convention ${ }^{16}$ ) $\lambda_{e f f} \equiv \frac{\kappa^{2}}{d}$.

In this work, we report on results obtained for $\lambda_{\text {eff }}=$ 64 for defects larger than the coherence length. As is typically the case for thin films, the new length scale $\lambda_{e f f}$ is considerably larger than $\lambda$. In fact, this choice of $\lambda_{e f f}$ is deep enough into the high $\lambda_{e f f}$ limit that, apart from scale factors in $E_{G L}$ and $\phi$, the results vary little as $\lambda_{\text {eff }}$ is increased.

To analyze the behavior of the vortex near the pinning center, we employ a mean field approach in which one minimizes $E_{G L}$ for a fixed vortex location to find $\psi, \vec{A}$, and the current $\vec{j}$. Our strategy for calculating $\psi$ and $\vec{A}$ self-consistently involves first holding $\phi$ fixed at some initial guess, and minimizing $E_{G L}$ with respect to $\vec{A}$ and $\vec{B}$. Next, we fix $\vec{A}$ and $\vec{B}$ and minimize with respect to $\phi$. These steps are iterated until changes in the variables become negligible. In the case of an external current, minor adjustments are needed which will be discussed later. We implement this self-consistent approach numerically by dividing the unit cell into a fine lattice of small unit cells. In this discrete scheme, $\phi(\vec{r})$ is replaced by $\phi_{i j}$ with ij specifying a grid point on a square lattice, while $\vec{A}_{i j}$ and $\vec{j}_{i j}$ are defined on nearest-neighbor links between the grid points. Derivatives in Eq. 1 are replaced by the corresponding finite differences. To model the defect array as accurately as possible, one desires a fine grid; we find that with a $128 \times 128$ mesh our results are well-converged with respect to the discretization.

To see how one minimizes $E_{G L}$ under the constraint of a specified vortex location, it is useful to write the current $\vec{j}$ in terms of the order parameter $\phi$ and the vector potential $\vec{A}$. By minimizing $E_{G L}$ with respect to the vector potential and employing a Maxwell equation one has

$$
\begin{aligned}
\vec{j} & =\frac{1}{2}\left[\phi^{*}\left(\vec{\nabla}_{\|} / i-\vec{A}\right) \phi+\phi\left(-\vec{\nabla}_{\|} / i-\vec{A}\right) \phi^{*}\right] \\
& =|\psi|^{2}\left(\vec{\nabla}_{\|} \theta-\vec{A}\right) .
\end{aligned}
$$

In Eq. 3 we have used for the order parameter $\phi=|\phi| e^{i \theta}$, and the gradient $\vec{\nabla}_{\|}=\left(\partial_{x}, \partial_{y}, 0\right)$. The familiar fluxoid quantization condition ${ }^{15}$ arises from the requirement that the order parameter be single valued, i.e. $\oint \vec{\nabla} \theta \cdot d \vec{s}=$ $2 \pi n_{v}$ with $n_{v}$ an integer. Hence, in terms of $\vec{j}$ and $\vec{A}$,

$$
\begin{aligned}
2 \pi n_{v}(i j) & =\oint\left(\vec{J} /|\phi|^{2}\right) \cdot d \vec{s}+\oint \vec{A} \cdot d \vec{s} \\
& =\oint\left(\vec{j} /|\phi|^{2}\right) \cdot d \vec{s}+\Phi_{B}
\end{aligned}
$$

In the second part of Eq. $4, \Phi_{B}$ is the total magnetic flux passing through the area of the contour, which we conveniently choose to be the small unit cell associated with the grid point $i j$, while $n_{v}(i j)$ is the total number of "fluxoid quanta" contained in the contour of integration ${ }^{15}$. It is through $n_{v}$ that the vortex location(s) in the full unit cell of the system may be fixed: $n_{v}=0$ except at the grid points where we wish to place a vortex, for which $n_{v}=1$. As discussed in the Appendix, one can easily calculate $\Phi_{B}$ and $\vec{A}$ self-consistently in the thin film limit from the current $\vec{j}$. Hence, armed with knowledge of $|\phi|$ and some specified realization of $n_{v}(i j)$, one can solve for $\vec{j}$ and $\vec{A}$ via Eq. 4 . Using the expression for the current given in Eq. 3 , one obtains $\vec{\nabla} \theta$; inserting $\vec{\nabla} \theta$ and $\vec{A}$ into Eq. 1 yields an expression depending only on $|\phi|$ and $\lambda_{e f f}$ which we minimize with respect to $|\phi|$ to obtain the order parameter modulus.

Our calculational method is easily generalized to include a supercurrent $\overrightarrow{j_{o}}$ flowing across the system, allowing the effects of a depinning current to be probed. This is accomplished by introducing a constant planar vector potential $\vec{a}_{o}$. $\vec{a}_{o}$ causes a shift in the current given by $\Delta \vec{J}=-|\phi|^{2} \vec{a}_{o}$.

In the above method, we have been careful to explicitly take into account the thin film geometry typical of experiments on nanoscale defect arrays. However, we note that this approach may easily be modified to handle the bulk case, in which the vortices resemble filaments of magnetic flux instead of pancake structures. We have performed a few such calculations, and find that for antidot systems the results obtained are quite similar to the large $\lambda_{e f f}$ results we report here. This may be understood in terms of 
the effective magnetic penetration depth for a thin film, $\lambda_{e f f}=\frac{\lambda^{2}}{d}$, which is typically much larger than the bulk value $\lambda^{16}$. This means that the energy stored in the magnetic field generated by the supercurrents is quite small, so that the fact that the field varies as one moves out of the plane has little impact on the state of the system. The resulting energy functional is thus nearly identical to the bulk three dimensional case in the large $\kappa$ limit, with columnar antidots and vortices.

\section{RESULTS}

\section{A. Zero Current}

At large vortex-defect separations, the core has the usual compact structure with supercurrents localized about it. Fig. 1 presents a perspective plot of $|\phi|$, as well as a vector plot of the currents. The distances shown are in units of the coherence length, $\xi$. Our choice of a unit cell with side spanning 20 coherence lengths and an antidot $5 \xi$ in size is typical of many of the nanoscale arrays studied experimentally. As the flux quantum nears the defect edge, it eventually reaches a critical distance $d_{c}$ (typically several $\xi$, with precise value depending on the dot size and shape), where there is a sudden dramatic change. Fig. 2 illustrates the situation after the transition: the vortex core has developed a string extending from the flux quantum position to the defect edge; simultaneously the current now encircles the vortex-defect pair, and the magnetic flux created by these currents spreads over a larger area. The string is energetically favorable because it allows the formerly dense current of the vortex to spread out (engulfing the defect in the process), thereby reducing the kinetic energy of the state.
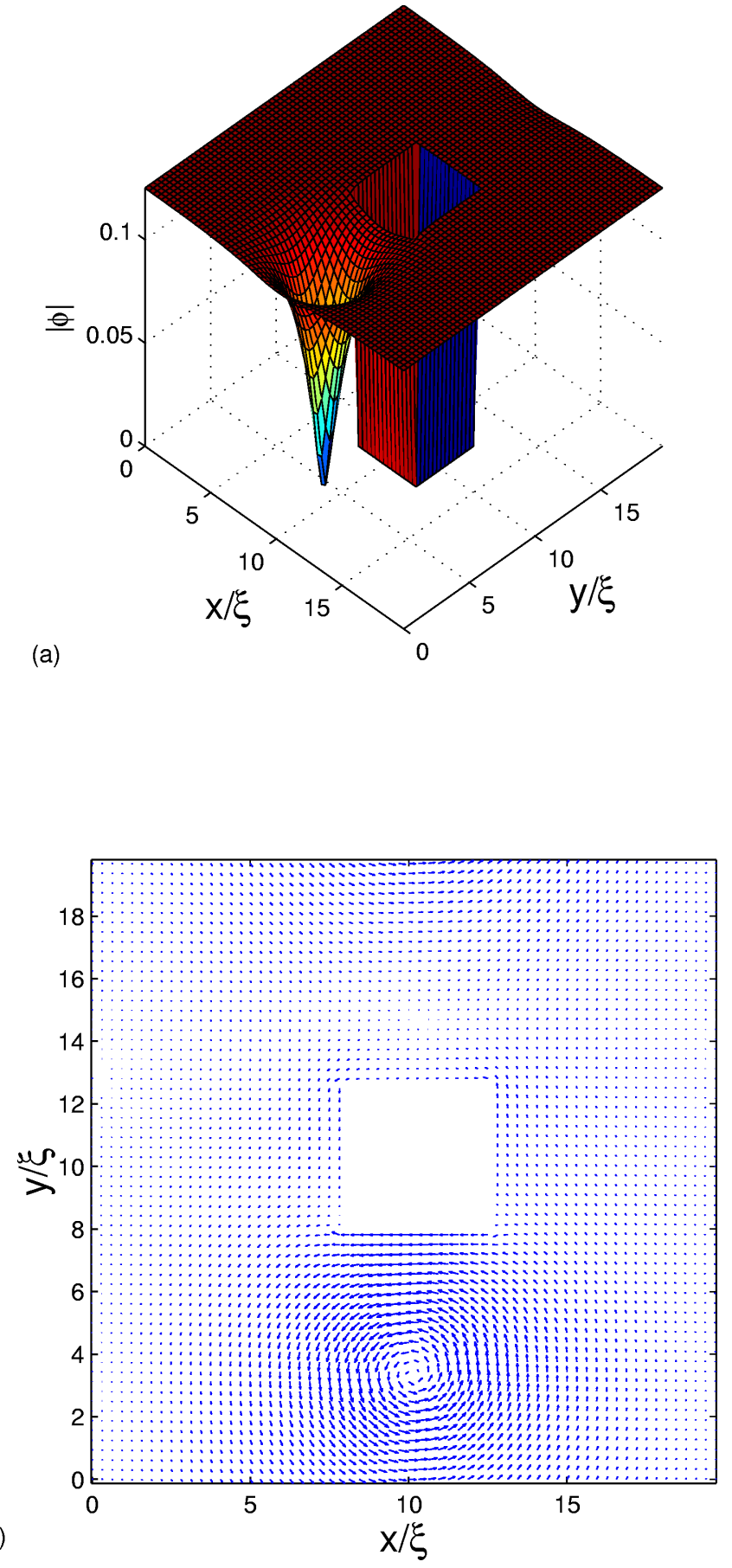

FIG. 1. A perspective plot of the order parameter modulus, $|\phi|$ (a) and current image (b) in a periodic antidot array just prior to the transition. Currents are localized about the vortex core, which has a compact structure. 


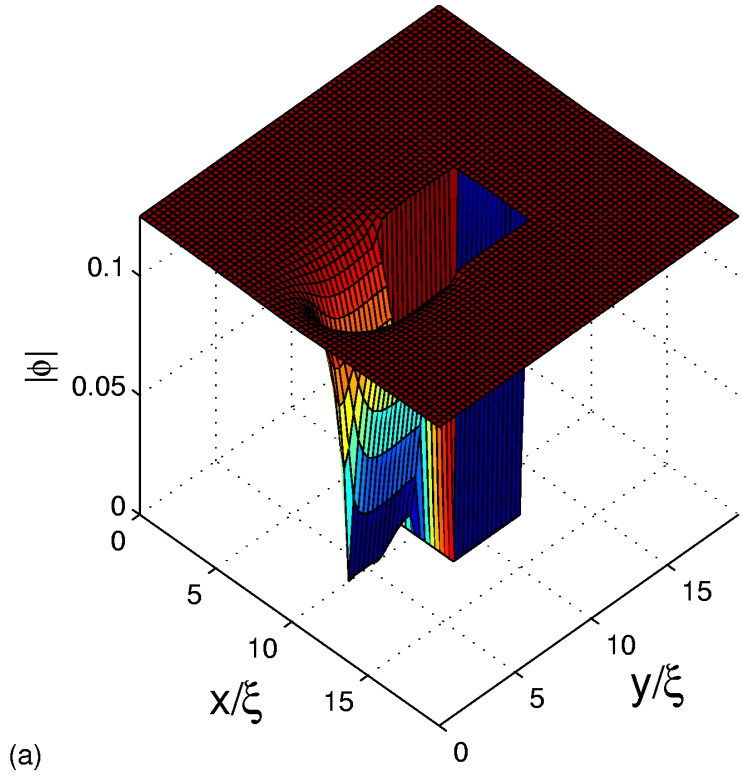

(a)

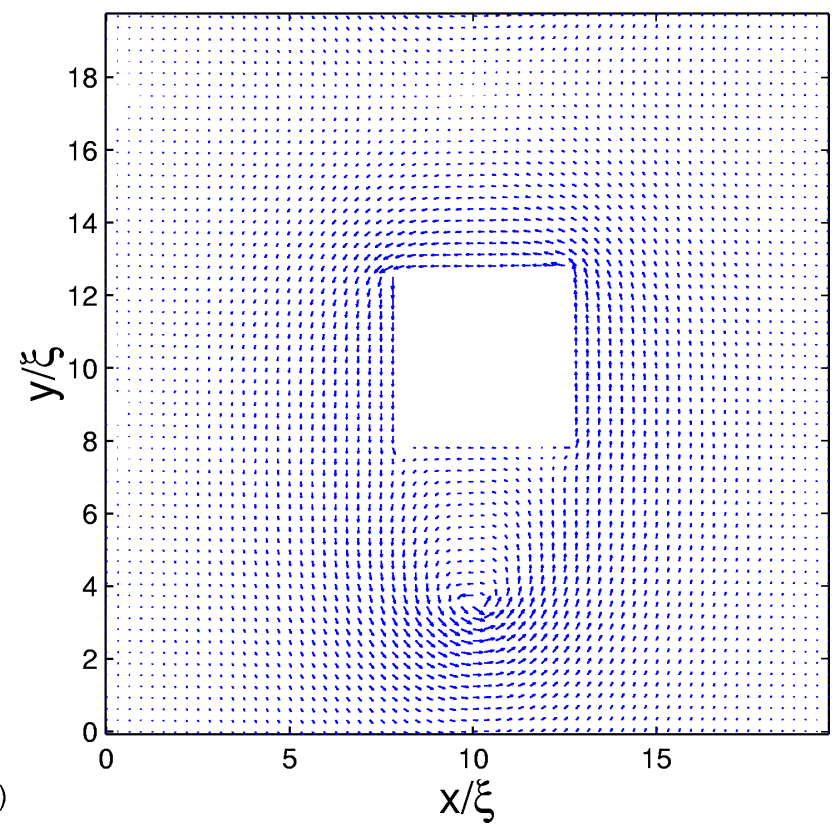

FIG. 2. Order parameter perspective plot (a) and currents (b) in the periodic array just after the transition. The currents circulate about the vortex-defect pair, and the vortex core has a string extending all the way to the defect edge.

When a net supercurrent flows across a superconductor, a Lorentz force is exerted on vortices; if these move in response energy is dissipated. In an artificial defect array, the driving force can be balanced by a pinning force given by the gradient of $E_{G L}$ with respect to the vortex position. Thus, one may regard $E_{G L}$ as the pinning potential. Fig. 3 shows this as a function of distance from the unit cell edge, which can be seen to have three distinct regions. For large separations, where the vortex core is compact, the pinning potential decreases relatively slowly. As the separation decreases it eventually crosses $d_{c}$ and the core-string structure appears. Hysteresis in the calculations, indicated with arrows, suggests that the transition is first order. However, as will be seen below, a finite current flowing across the unit cell changes the character of the transition, rendering it continuous. Below the transition one observes nearly linear behavior of the pinning potential, suggesting that the string carries an energy proportional to its length. The third region is announced by a discontinuous jump as the vortex is absorbed by the defect, followed by a perfectly flat region inside the defect. This abrupt jump is due to a sudden transformation of the order parameter. Immediately prior to absorption, constant Cooper pair density contours near the flux quantum have a semicircular profile. As the vortex enters the defect, this "semi-vortex" vanishes, removing a finite amount of energy for an infinitesimal change in the vortex position. In later discussion, the $\Delta_{e j}$ shall be used to refer to this jump in energy.

\section{B. Finite Current}

When a net current flows across the superconducting film, a Lorentz force is exerted on the current distribution by the external magnetic field. This nonconservative force, given by $\frac{1}{c} \boldsymbol{\Phi}_{\mathbf{o}} \times \mathbf{J}^{15}$, acts in opposition to $\vec{\nabla} E_{G L}$. In terms of our units, the magnitude of the Lorentz force is $4 \pi j^{\prime}$, where $j^{\prime}$ is the net current magnitude (In CGS units, $\left.J=\frac{\hbar c^{2}}{8 \pi d e \xi^{3}} j^{\prime}\right) . E_{G L}$ also depends on $j^{\prime}$. As will be seen, this dependence is particularly manifest near the depinning threshold.

Figure 4 indicates the evolution of $E_{G L}$ as the current is increased. As the two graphs in Fig. 4 reveal, even for currents a reasonable fraction $(17 \%$ and $33 \%$ respectively) of the bulk critical current $J_{c}, E_{G L}$ is similar to that of the zero-current case, except that the breadth of the linear region increases with drive current. Ultimately, as can be seen in the potential curve corresponding to the larger current, the linear behavior extends to either edge of the unit cell. This suggests there is a range of net current in which the vortex core always exists as a stringlike structure. Another important feature is the fact that the jump in energy when the fluxoid exits the dot (which we call the "ejection barrier" $\Delta_{e j}$ ) does not appreciably diminish as the drive current $j^{\prime}$ is increased. An interesting observation, apparent in Figs. 4, is that the Lorentz force increases with increasing current while the slope of the conservative part of the pinning potential remains nearly constant. Eventually, the Lorentz force exceeds the slope of $E_{G L}$ vs. fluxoid position before the jump $\Delta_{e j}$ has been eliminated. This suggests that the ejection of the vortex 
with increasing drive current will be sudden at low temperatures, i.e., once the flux quantum is able to exit the dot, the string tension will not be sufficiently strong to keep the vortex bound. We will see below that this is indeed the case. At higher temperatures, when $T>\Delta_{e j}$, one expects to see depinning for somewhat lower drive currents.

Depinning occurs when the net current is made large enough to eliminate $\Delta_{e j}$. This occurs suddenly as one increases the current beyond a certain threshold, $j_{d p}^{\prime}$. As Figure 5 indicates, the net supercurrent drops abruptly; presumably the remainder of the drive current appears in resistive channels. The fact that the solutions to the Ginzburg-Landau theory have a maximum possible value $j^{\prime}$ even for large $a_{o}^{x}$ is highly analogous to what happens in thin superconducting wires and films ${ }^{15}$. As in those cases, this maximum current should be identified as a critical current; in our case, it is the depinning current $j_{d p}^{\prime}$. As illustrated in Figure 6 and Figure 7 , the depinning transition is marked by the sudden emergence of a core string. The string, a normal region which blocks supercurrents, accounts for the sudden drop in the supercurrent flow across the unit cell. For the system parameters we have chosen, the depinning current, $j_{d p}^{\prime}$ is $62 \%$ of the bulk critical current $j_{c}^{\prime}$.

Strikingly, currents capable of depinning the vortex do so without forming a flux quantum that has a compact core. In fact, as the vortex moves across the unit cell, its core always has a stringlike structure. As the flux quantum is driven from the pinning defect, a core "string" forms connecting the vortex to the edge of the pinning defect. Even before the flux quantum emerges from the pinning defect, this string is already partly formed. As the vortex traverses the unit cell toward the neighboring defect, the core string lengthens. This continues until the flux quantum reaches the unit cell edge, where the magnetic flux and currents unwrap from the pinning defect and engulf the adjacent defect. Figure 8 and Figure 9 illustrate this transition. Figure 8 displays the order parameter modulus and current patterns just prior to the transition, while Figure 9 portrays the system just after the currents and flux have surrounded the neighboring defect.

We conclude this section by noting that the depinning process found for typical antidot arrays is markedly different than previous expectations, which commonly assume that in depinning the vortex takes a form similar to Figure 1. We expect the latter scenario to hold when the interdot separation is very large compared to the coherence length $\xi$. The different forms of depinning might be detectable in voltage noise experiments, which are analogous to shot noise experiments for electrons in conductors. When the vortices are small and compact, it is natural to suppose they will act as particles and, when depinned, yield a broadband noise proportional to the voltage across the system. If one increases the temperature and corresponding coherence length, the string depinning behavior described above must eventually apply. This eliminates short wavelength and presumably high frequency dynamics from the system. We thus expect a suppression of high frequency noise to accompany the transition into string-like depinning.

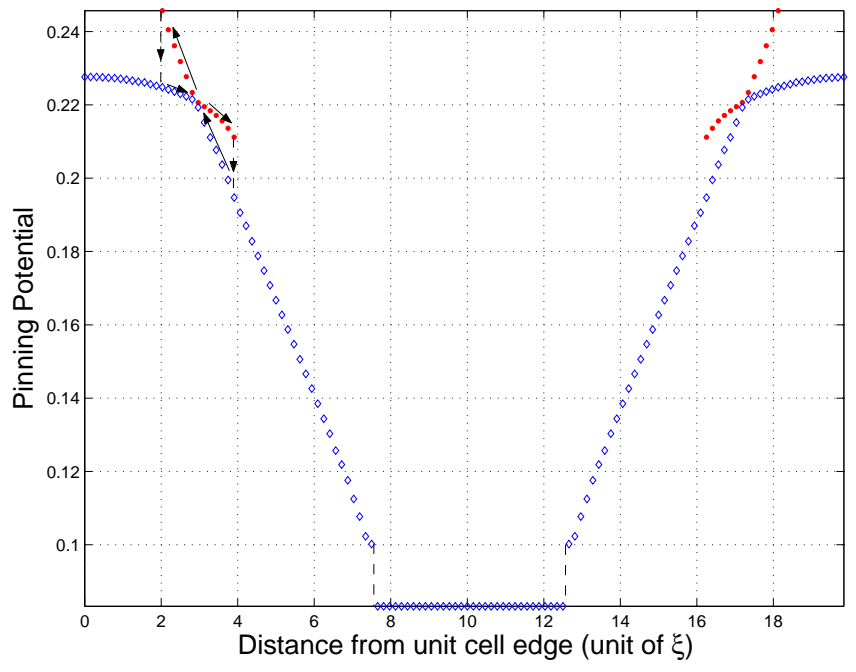

FIG. 3. Plot of the pinning potential showing broad linear regions and a gap in the potential at the defect boundary. The horizontal axis measures the distance of the vortex from the edge of the unit cell. Arrows indicate hysteresis in the calculation, a hallmark of a first order transition. 

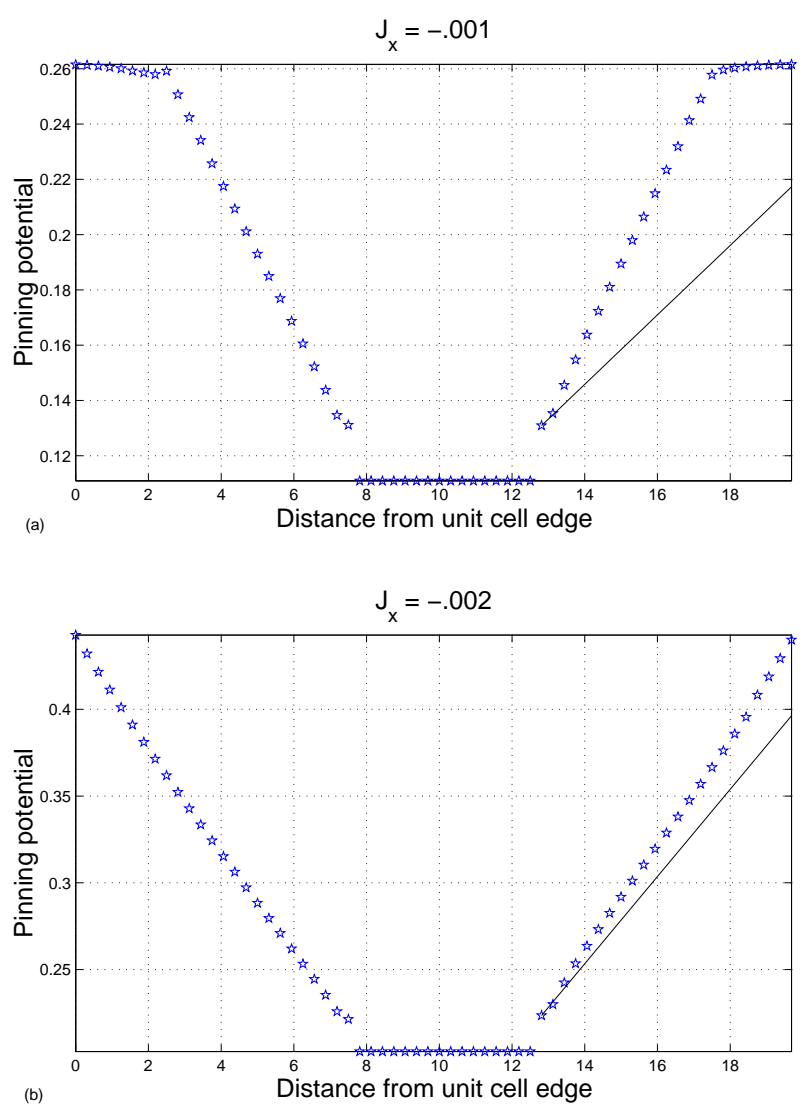

(b)

FIG. 4. Pinning potentials for $J_{x}=-.001$ (a) and $J_{x}=-.002(\mathrm{~b})$ The exit barrier for the flux quantum does not diminish despite increasing drive current. The slope of the dark line in both plots is the Lorentz force.
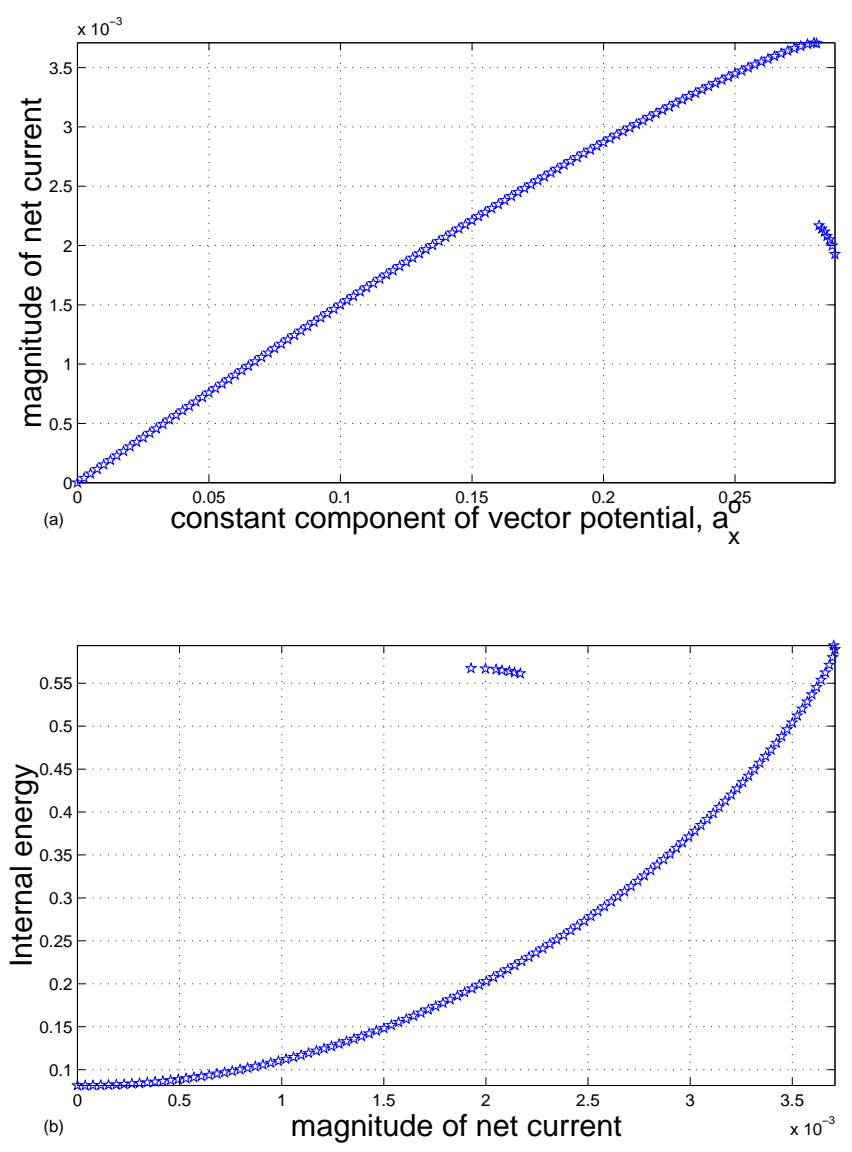

FIG. 5. Plot of net current as a function of $a_{x}^{0}$ (top) and Ginzburg-Landau energy versus the current (bottom) 


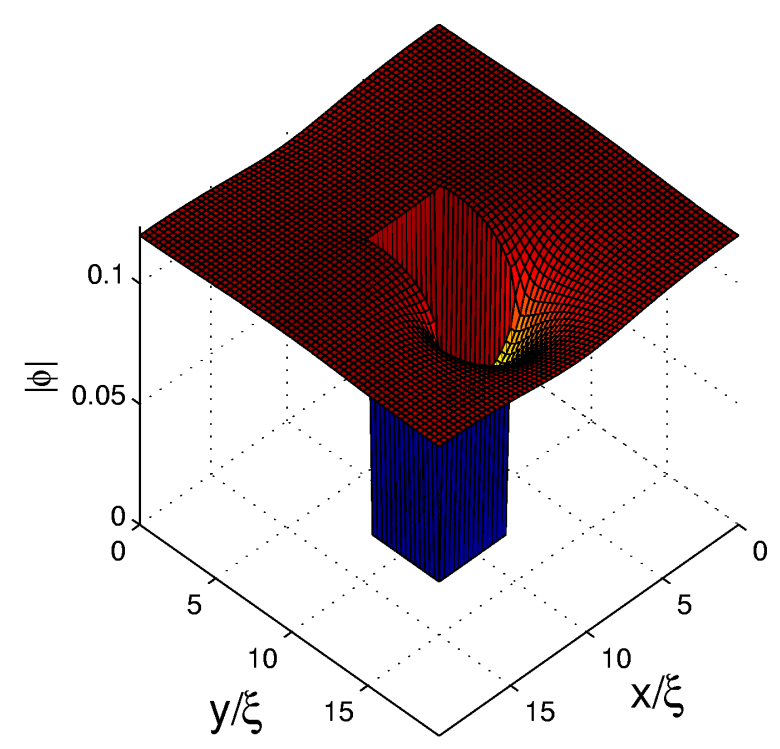

(a)

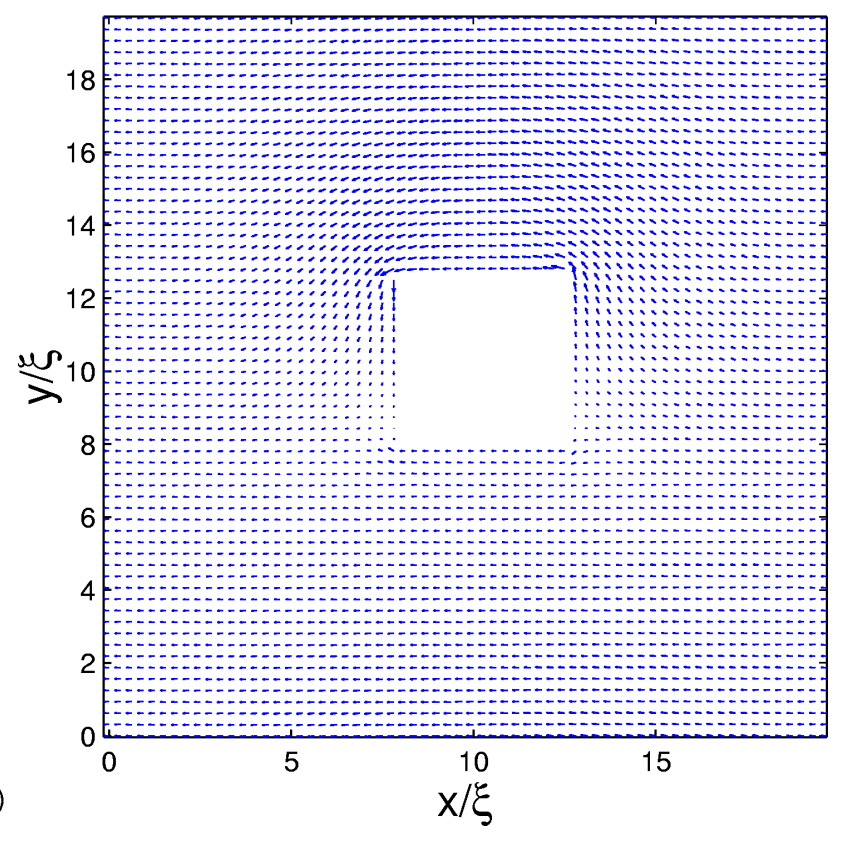

FIG. 6. A perspective plot of the order parameter modulus, $|\phi|(\mathrm{a})$ and current image (b) in a periodic antidot array just prior to the transition. A core string has not appeared.

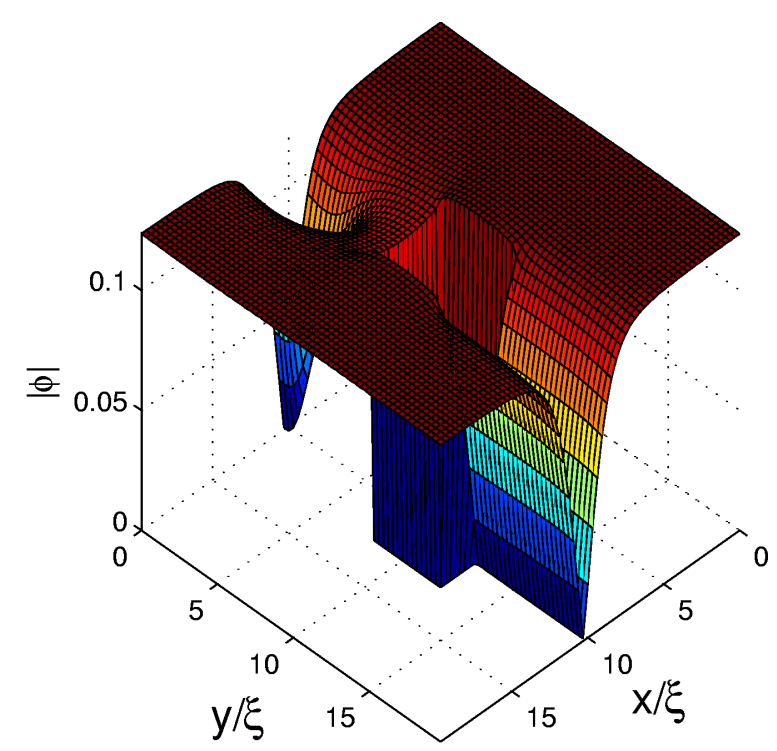

(a)

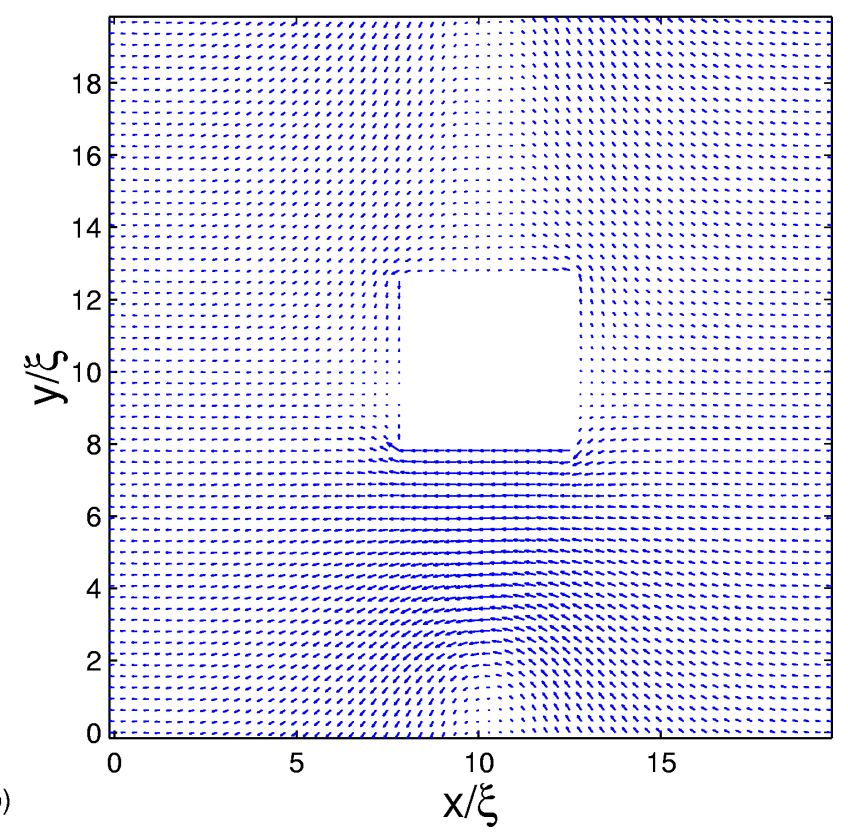

FIG. 7. A perspective plot of the order parameter modulus, $|\phi|$ (a) and current image (b) in a periodic antidot array with core string, just beyond depinning 


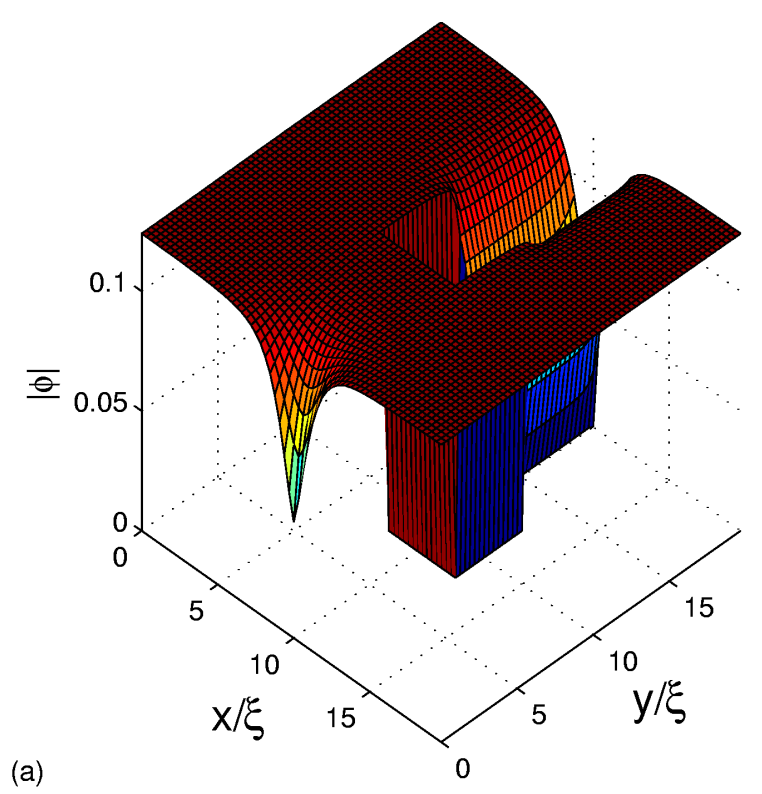

(a)

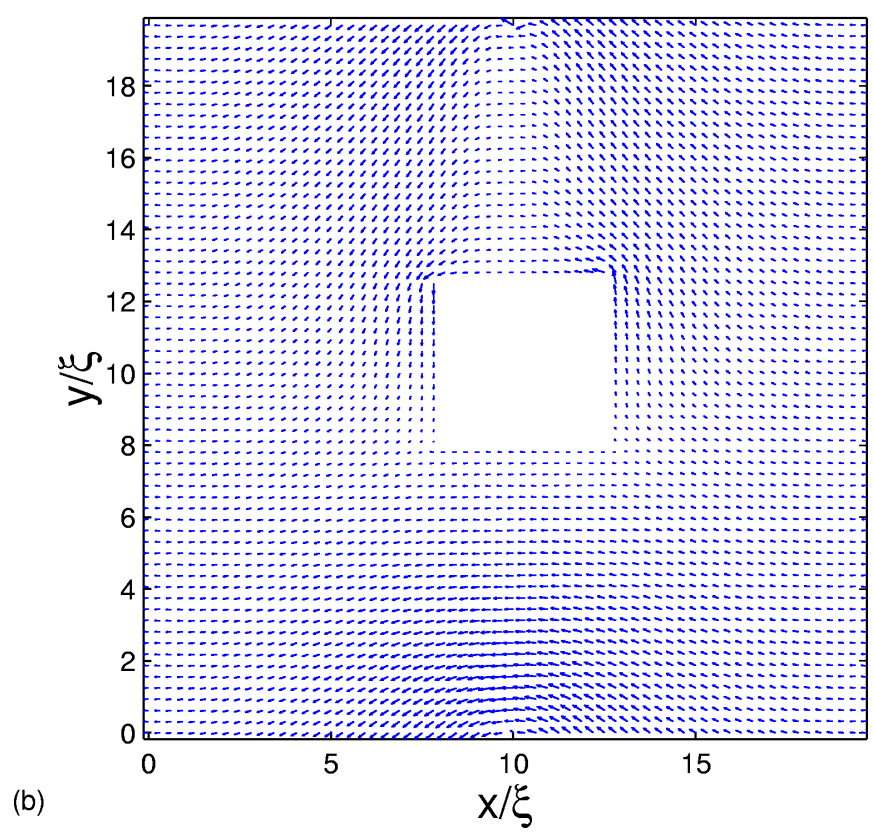

FIG. 8. A perspective plot of the order parameter modulus, $|\phi|(\mathrm{a})$ and current image (b) in a periodic antidot array just prior to the transition. The core string is directed away from the pinning defect
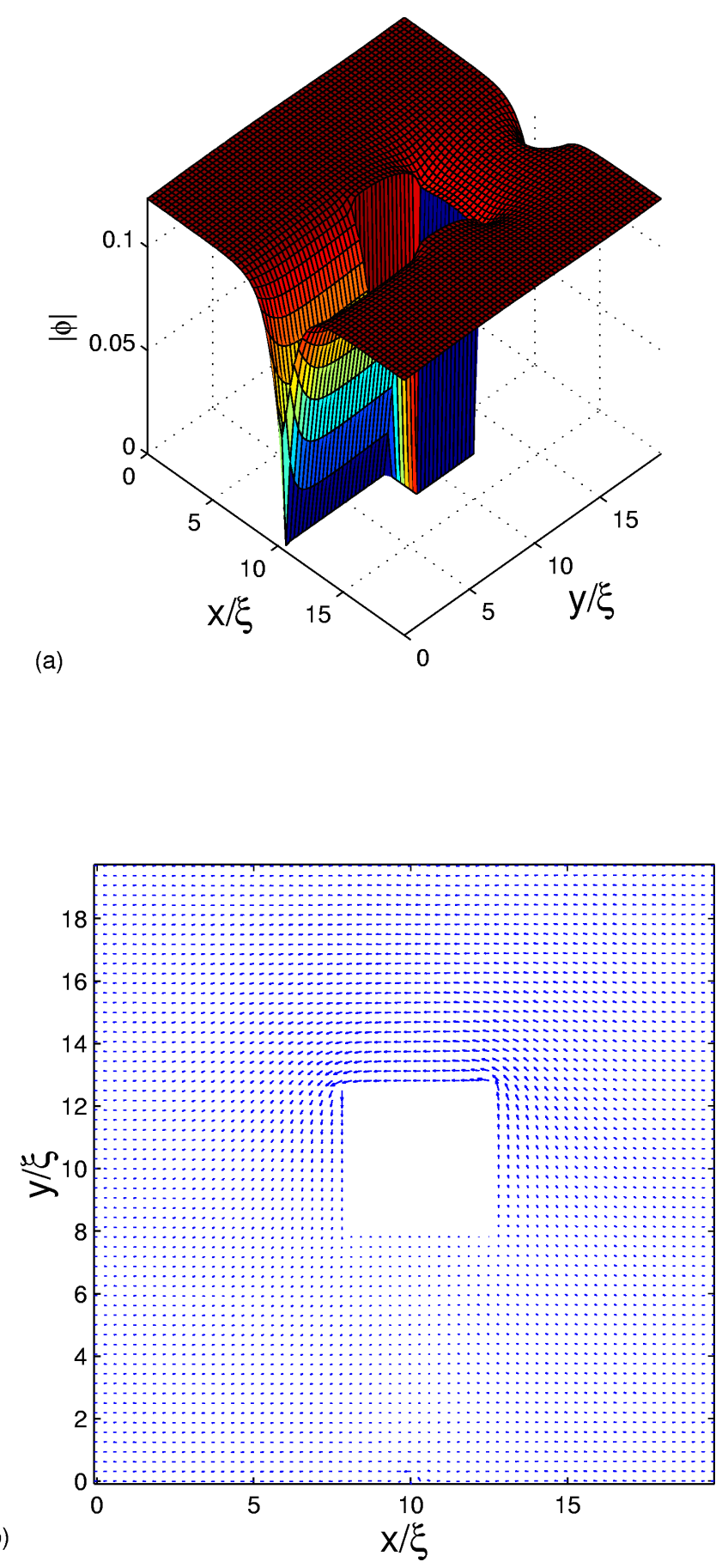

FIG. 9. A perspective plot of the order parameter modulus, $|\phi|$ (a) and current image (b) in a periodic antidot array just after the "unwrapping" transition. The core string is attached to the adjacent "destination" defect. Currents have moved from the pinning defect and encircle the "destination" defect. 


\section{EXPERIMENTAL IMPLICATIONS}

Beyond the voltage noise behavior described above, the profile of the pinning potential shown in Fig. 3 suggests a set of measurements one might perform to seek an experimental signature of the unique pinning phenomena discussed above. Past the depinning threshold, core strings moving among the antidots form linear channels which might appear as an anisotropy signal in neutron scattering measurements. Another possible test would involve working at temperatures large compared to $\Delta_{e j}$, but small compared to the energy at the unwrapping transition, as in Fig. 2. In this situation, an AC driving force on the vortices, of magnitude small enough to leave them pinned by the strings (as might be provided in a vibrating reed experiment ${ }^{17}$ ) would allow the vortices to "rattle around" in the linear part of the pinning well illustrated in Fig. 3 and produce losses, whereas a DC force of equal magnitude would be dissipationless. Upon lowering of the temperature the vortices would be captured by the defects, leading to lossless supercurrents for both AC and DC driving forces. An observation of these effects would yield indirect confirmation of the form of the pinning potential we find. Something like this may recently have been observed ${ }^{17}$.

Finally, a consequence of our results is that the depinning current should increase sharply as $T$ is lowered below $\Delta_{e j}$. For $T>\Delta_{e j}$, vortices will be thermally excited above the ejection barrier, and depinning can occur when the slope of $E_{G L}$ versus fluxoid position is equal to the Lorentz force. For $T \ll \Delta_{e j}$ the current must be large enough to eliminate the ejection barrier to depin the vortex ${ }^{19}$.

Another interesting possibility is that a unique thermal depinning may occur as the temperature is increased in the regime of linear pinning. The presence of a string suggests that this may carry entropy at finite temperature, much as is the case of polymers. This entropy is proportional to the string length and temperature, and at high enough temperatures may overwhelm the energy per unit length found in our mean-field calculations. In analogy with polymer behavior ${ }^{18}$, this leads to unbounded growth of the string and effective depinning of the vortex. However, it is not clear whether the string remains sufficiently well-defined at the temperatures necessary for proliferation that the polymer analogy remains valid up to the transition. Further research into this possibility is currently underway.

\section{SUMMARY}

Using Ginzburg-Landau theory, we have given a detailed treatment of the microscopic aspects of pinning phenomena in nanoscale periodic arrays. Strikingly, we see an apparent first order transition involving the creation of a string connecting the vortices to the defect, and an accompanying abrupt transformation of the supercurrent and the magnetic field it generates. The string configuration leads to a region of linear pinning. Absorption of the vortex by the antidot is marked by a jump in the pinning potential, $\Delta_{e j}$. Depinning occurs abruptly when the drive current exceeds $J_{d p}$. Various aspects of the pinning potential should be observable in experiment.

Acknowledgments - The authors would like to thank L. E. DeLong, S. B. Field, and J. B. Ketterson for useful discussions. This work was supported by NSF Grant No. and DMR-0108451.

\section{APPENDIX A: CALCULATION OF $\Phi_{B}$}

In this Appendix, we outline the calculation of the magnetic flux density in terms of the supercurrents in the thin film. The lattice variables mentioned in this work are discrete approximations of continuum quantities. For the sake of brevity, however, the analysis in this Appendix is given in the continuum limit. The calculation sketched here is largely parallel to an earlier work in the context of the London theory ${ }^{16}$.

We adopt units for which the current $\overrightarrow{J^{\prime}}$ satisfies $\overrightarrow{J^{\prime}}=$ $\vec{\nabla} \times \vec{B}$. Taking advantage of the fact that $\vec{\nabla} \cdot \vec{A}=0$ in the London gauge, we find that

$$
\overrightarrow{J^{\prime}}=-\nabla^{2} \vec{A}
$$

Fourier transforming Eq. A1 yields

$$
\overrightarrow{J_{\mathbf{k}}^{\prime}}=-k^{2} \overrightarrow{A_{\mathbf{k}}}
$$

Defining $k_{\|}^{2} \equiv\left(k_{x}^{2}+k_{y}^{2}\right)$, we have

$$
J_{\mathbf{k}}^{\prime x, y}=-\left(k_{z}^{2}+k_{\|}^{2}\right) A_{\mathbf{k}}^{x, y}
$$

To express $\overrightarrow{J_{\mathbf{k}}^{\prime}}$ in terms of the two dimensional currents flowing in the plane of the film one uses the fact that $\overrightarrow{J^{\prime}}$ is essentially constant in the $\hat{z}$ direction in the thin film limit. Hence, we have

$$
J^{\prime} x, y=\left\{\begin{array}{c}
J_{2 d}^{\prime} x, y,-\frac{d}{2} \leq z \leq \frac{d}{2} \\
0 \text { elsewhere }
\end{array}\right\}
$$

As the film thickness diminishes to zero, we obtain

$$
J^{\prime} x,\left.y\right|_{d \rightarrow 0}=\delta(z) j_{2 d}^{\prime x, y}
$$

Fourier transforms equation A5 yields

$$
J_{\mathbf{k}}^{\prime} x, y=\frac{1}{L_{z}} j_{\mathbf{k}_{\|}}^{\prime} x, y
$$

In Eq. A6 above, $\mathbf{k}_{\|}$denotes the plane wave vector. We infer from Eqs. A3, A6 that 


$$
A_{\mathbf{k}}^{x, y}=-\frac{1}{L_{z}\left(k_{z}^{2}+k_{\|}^{2}\right)} j_{\mathbf{k}_{\|}{ }^{\prime}, y}
$$

To obtain $\left.A^{x, y}\right|_{z=0}$, and $\left.B^{z}\right|_{z=0}$, an inverse Fourier transformation of Eq. A7 is needed. One finds that

$$
\begin{aligned}
\left.A^{x, y}\right|_{z=0} & =\iint L_{x} L_{y} \int \frac{-(2 \pi)^{-3} j_{\mathbf{k}_{\|}{ }^{\prime}, y}}{\left(k_{z}^{2}+k_{\|}^{2}\right)} d^{3} k \\
& =\frac{L_{x} L_{y}}{2(2 \pi)^{2}} \iint \frac{-j_{\mathbf{k}_{\|}{ }^{\prime}, y}}{k_{\|}} d k_{x} d k_{y}
\end{aligned}
$$

To obtain $\left.B^{z}\right|_{z=0}$, we use the fact that

$$
\left.B^{z}\right|_{z=0}=-\left.\frac{\partial}{\partial y} A_{x}\right|_{z=0}+\left.\frac{\partial}{\partial x} A_{y}\right|_{z=0}
$$

In Fourier space, one has

$$
\left.B_{\mathbf{k}_{\|}}^{z}\right|_{z=0}=-\left.k_{y} A_{\mathbf{k}_{\|}}^{x}\right|_{z=0}+\left.k_{x} A_{\mathbf{k}_{\|}}^{y}\right|_{z=0}
$$

Hence, we find that

$$
\left.B^{z}\right|_{z=0}=\frac{L_{x} L_{y}}{2(2 \pi)^{2}} \iint \frac{\left(k_{y} j_{\mathbf{k}_{\|}^{\prime}}{ }^{x}-k_{x} j_{\mathbf{k}_{\|}{ }^{\prime}}\right)}{k_{\|}} d k_{x} d k_{y} .
$$

To calculate $\Phi_{B}^{i j}$, the magnetic flux passing through the small unit cell at the point $i j$, we integrate $\left.B^{z}\right|_{z=0}$ over the area of the small square. In the discretization scheme employed in this work, we use the approximation

$$
\Phi_{B}^{i j}=\left.a^{2} B_{i j}^{z}\right|_{z=0},
$$

where $a^{2}$ is the area of the small unit cell.

${ }^{1}$ K. Harada et al. Science 274, 1167 (1996).

${ }^{2}$ M. Baert, V. V. Metlushko, R. Jonckheere, V. V. Moshchalkov, and Y. Bruynseraede. Phys. Rev. Lett. 74, 3269 (1995).

${ }^{3}$ V. V. Moshchalkov et al, Phys. Rev. B 54, 7385 (1996).

${ }^{4}$ A. Tonomura et al, Nature 397, 308-309, (1999)

${ }^{5}$ A. Tonomura et al, Nature 412, 620-622, (2001)

${ }^{6}$ S. B. Field et al, cond-mat/0003415.

7 T. Matsuda, K. Harada, H. Kasai, O. Kamimura, and A. Tonomura, Science 271, 1393 (1996).

${ }^{8}$ C. Reichhardt, A. B. Kolton, and D. Dominguez, and N.Gronbech-Jensen, Phys. Rev. B 64, 134508 (2001); C. Reichhardt, G. T. Zimanyi, and N. Gronbech-Jensen, Phys. Rev. B 64, 014501 (2001).

${ }^{9}$ G.W. Crabtree, D.O. Gunter, H.G. Kaper, A.E. Koshelev, G.K. Leaf, and V.M. Vinokur, Phys. Rev. B 61, 1446 (2000).
${ }^{10}$ F. Nori, Science 271, 1373 (1996); C. Reichhardt, C. J. Olson, and F. Nori, Phys. Rev. Lett. 78, 2648-2651 (1997); M.C. Cha and H.A. Fertig, Phys. Rev. Lett. 80, 3851 (1998).

11 A. I. Buzdin, Phys. Rev B 47, 11416 (1993).

12 G. S. Mkrtchyan and V. V. Shmidt, Sov. Phys. JETP 34, 195 (1972).

${ }^{13}$ I. B. Khalfin and B. Ya. Shapiro, Physica C 207, 359 (1993).

14 Sa-Lin Cheng, D.J. Priour Jr, and H.A. Fertig, Phys. Rev B 65, 024503 (2002).

${ }^{15}$ M. Tinkham, Introduction to Superconductivity, Second edition, McGraw-Hill, Inc., New York, (1996).,

16 J. Pearl, Appl. Phys. Lett. 5, 65 (1964); P.G. de Gennes, Superconductivity of Metals and Alloys Addison-Wesley, New York, (1989).

17 J. Zhang, L.E. De Long, V. Majidi, and R.C. Budhani, Phys. Rev B 53, R8851 (1996).

${ }^{18}$ C. Vanderzande, Lattice Models of Polymers, Cambridge University Press, Cambridge (1998).

${ }^{19}$ However, it should be noted that the change in depinning current is of order $50 \%$, so that this phenomenon may be difficult to sort out from other temperature dependence entering through the coherence length and other GL parameters. 